\title{
Energy-Efficient Cluster-Head Rotation in Beacon-Enabled IEEE 802.15.4 Networks
}

\author{
Hamidreza Tavakoli ${ }^{1}$, Jelena Mišić ${ }^{2}$, Senior Member, IEEE, \\ Vojislav B. Mišić ${ }^{2}$, Senior Member, IEEE, and Majid Naderi ${ }^{1}$ \\ ${ }^{1}$ Iran University of Science and Technology, Tehran, Iran \\ ${ }^{2}$ Ryerson University, Toronto, ON, Canada
}

\begin{abstract}
Wireless sensor networks that use slotted, beaconenabled IEEE 802.15.4 standard are often implemented using clustering with randomized sleep of ordinary nodes to extend the network lifetime. To reduce the imbalance of power consumption between cluster-head and ordinary nodes, we propose a fair and efficient cluster-head rotation protocol with virtually zero overhead and no dead time. Through Markov chain modeling and probabilistic analysis, we show that the proposed approach achieves extremely good balance of nodes' lifetime and optimal network lifetime in maximin sense, outperforming other approaches that use TDMA access.
\end{abstract}

Index Terms-Energy-efficient clustering, cluster-head rotation, IEEE 802.15.4, network lifetime, wireless sensor networks

\section{INTRODUCTION}

Energy efficiency at both individual node- and network level is probably the most important objective for the design of wireless sensor networks [36]. Energy efficiency may be achieved by applying techniques that minimize overhead and balance the energy consumption of individual nodes to the highest extent possible [37]. Two among the techniques often used to achieve these objectives are sleep and clustering.

Sleep refers to low power operation of sensor nodes whenever they have no data to send, which reduces energy expenditure. However, in order to maintain the application-specified rate of information flow, hereafter referred to as sensing reliability $R$ [2], the network should have redundant sensors - i.e., more sensors than the minimum needed to achieve $R$. Further energy savings can be achieved by adjusting the activity patterns of individual nodes [34].

Clustering refers to the partitioning of the network into groups or clusters with a designated leader or cluster-head (CH) [8], as shown schematically in Fig. 1. Individual sensor nodes send their data to the base station (BS) in a multihop manner, with one or more $\mathrm{CH}$ nodes as intermediaries. If clusters operate on separate frequency channels, contention among nodes and losses thus incurred are reduced. Unfortunately, clustering results in excessive energy expenditure of $\mathrm{CH}$ nodes which must remain active most, or even all of the time, while ordinary nodes need to be active only while they send their data. The balance is commonly restored by rotating (i.e., periodically re-assigning) the $\mathrm{CH}$ role to different nodes [30]. In addition, $\mathrm{CH}$ selection is often accompanied by reclustering (i.e., re-formation of clusters), during which regular sensing is partially or completely suppressed [6].

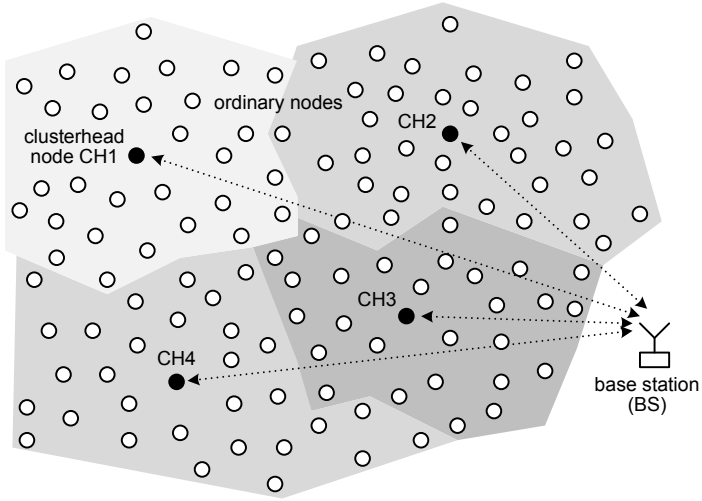

Fig. 1. Topology of a clustered WSN.

A number of clustering-based protocols have been described over the years [17], [30], [41]; most of them are based on some variant of TDMA-based medium access. However, the design of an optimal TDMA schedule requires significant computational overhead [9], [22], [35], and efficient implementations necessitate tight synchronization of node clocks [6].

The IEEE 802.15.4 low data rate wireless personal area network (LR-WPAN) technology has been widely used in recent years [20], [28]. It provides good scalability and supports both clustering and low power operation, which is why it has been used to implement wireless sensor networks in areas as diverse as intelligent buildings [7], smart grid [14], and healthcare [24]. The default medium access protocol in IEEE 802.15.4 is CSMA-CA, but contention-free access is possible through the so-called Guaranteed Time Slots (GTSs) [20]. CSMA-CA does suffer from packet collisions and ensuing retransmissions, but it also offers much better scalability compared to the TDMA schedule-based protocols.

In this paper, we describe an efficient approach to clustering in which $\mathrm{CH}$ selection is based on the remaining energy of the nodes, while $\mathrm{CH}$ rotation is achieved with minimum possible overhead by using the facilities provided by the IEEE 802.15.4 LR-WPAN protocol. Non-CH nodes employ random sleep between data transmissions which balances the power consumption of individual nodes and minimizes the likelihood of collisions [26]. Additional energy savings are provided by opportunistic sleep of $\mathrm{CH}$ nodes. Clustering is performed only during the initial setup, and cluster membership does not change during subsequent operation. In this manner, dead time 
is eliminated and clustering overhead is minimized.

Our analysis shows that the different aspects of the approach synergistically contribute to balancing the lifetimes of individual nodes with very small variability. If the network lifetime is defined as the time period during which all nodes that were present when the network started operation are alive, equalizing nodes' lifetime actually maximizes the minimum node lifetime and, thus, optimizes the network lifetime in the minimax (or, more precisely, maximin) sense [16].

We also show that the use of CSMA-CA offers improved energy efficiency compared to a contention-free approach that uses the GTS facility of the IEEE 802.15.4 standard.

The paper is organized as follows: after an overview of related work in Section II, we give a brief overview of IEEE 802.15.4 operation in Section III. Operation of the clustered network is presented in Section IV, while the cluster-head handover is discussed in Section V. Section VI presents and discusses numerical performance results, including the modification of $\mathrm{CH}$ node operation that substantially improves network lifetime. Finally, Section VII concludes the paper. An Online Supplement presents the detailed analytical model, built using probabilistic techniques and Markov Chain modeling, for energy consumption of the network that operates using the proposed mechanisms.

\section{RELATED WORK}

Clustering has been used in many algorithms and protocols proposed in the literature. LEACH [17] has been one of the first protocol to employ clustering and $\mathrm{CH}$ rotation. It assumes that all nodes can communicate with the BS and uses clustering to reduce power consumption by limiting the transmission range. LEACH uses TDMA schedule for individual node transmissions, under the assumption that a node always has data to send at the time of its scheduled transmission.

Many variations of the LEACH protocol have been described, including centralized $\mathrm{CH}$ selection by the BS [18], deterministic rather than random selection of $\mathrm{CHs}$ [15], and clustering that takes transmission distances into account [41].

Formation of clusters may be based on random choice between $\mathrm{CH}$ nodes, possibly including the impact of distance to the $\mathrm{CH}$ candidates (as measured by received signal strength) [17]. In multi-hop networks, another factor to consider is the distance to the $\mathrm{BS}$, as clusters and $\mathrm{CH}$ nodes closer to the BS have higher amounts of traffic and, consequently, higher energy consumption [31].

Many authors have used remaining node energy as the main criterion for selecting the next $\mathrm{CH}$ [11], [15], [40], although other criteria such as the age of the $\mathrm{CH}$ [17], amount of traffic, number of neighbors [5], and even density of sensor nodes [29] have been used as well.

All approaches that use TDMA require extensive information exchange between the nodes themselves or between the nodes and the BS, and thus impose considerable overhead and a 'dead time' during which regular data communication is slower or even completely suspended. The overhead is even higher if $\mathrm{CH}$ elections involve re-clustering, i.e., repeated partitioning of nodes into clusters. To combat this overhead, [23] restricts clustering to nodes in close proximity of each other, which tends to reduce communication costs. In the method described in [19], all nodes (including current $\mathrm{CH}$ nodes) take part in the $\mathrm{CH}$ election, but only nodes that exceed an energy threshold are eligible for the $\mathrm{CH}$ role.

However, a simpler approach to cluster-head selection is possible if we resort to probabilistic $\mathrm{CH}$ rotation which has been shown to improve the lifetime of the network compared to the deterministic rotation with a predefined period [30].

A clustering approach using an IEEE 802.15.4 network in the cluster-tree topology was described in [1]. In this approach, $\mathrm{CH}$ selection is performed within the AODV routing protocol, using a combination of remaining node energy, number of neighboring nodes, and number of hops to the root of the tree (i.e., the distance to the BS) as the criterion for $\mathrm{CH}$ selection. Minimization of energy consumption in a multi-hop scenario was also described in [3], but their approach focused on adapting the protocol parameters, rather than network topology, to reach that objective.

Randomized sleep is one of the most popular mechanisms to achieve energy efficiency [34], [36]. A distributed algorithm, based on the probabilistic Gur game automata, that aims to maintain the information rate received at the $\mathrm{BS}$ at the level required by the sensing application was described in [21], although sensor nodes never go to low power sleep since they need to listen to the BS at all times. A similar approach using a simple probabilistic automaton was described in [10]. However, neither of these approaches uses IEEE 802.15.4 technology, unlike the activity management approach described in [26] which is fully integrated with the IEEE 802.15.4 MAC protocol; our approach to sleep management uses this work as the foundation.

\section{IEEE 802.15.4 MAC LAYER OPERATION}

Wireless sensor networks that use IEEE 802.15.4 LRWPAN communication technology commonly operate in beacon-enabled, slotted CSMA-CA mode, in which individual nodes form clusters under the control of their respective coordinators or cluster-heads. In each cluster, channel time is divided into superframes bounded by beacon frames transmitted by $\mathrm{CH}$ nodes. The structure of a superframe is shown in Fig. 2. The duration of the active portion of the superframe, $S D$, is determined through the so-called superframe order $S O$ as $S D=48 \times 2^{S O}$, expressed in unit backoff periods. Similarly, the interval between successive beacons (beacon interval) is determined by the so-called beacon order $B O$ as $B I=48 \times 2^{B O}$. The two variables satisfy the condition $0 \leq S O \leq B O \leq 15$ [20]. When the cluster operates in the unlicensed Industrial, Scientific, and Medical (ISM) band at $2.4 \mathrm{GHz}$, a unit backoff period lasts for $320 \mu$ s and contains 10 bytes, giving a raw data rate of $250 \mathrm{kbps}$ [20].

All communications in a cluster take place during the active portion of the superframe. Transmission of a packet in the uplink direction, i.e., from the node to the coordinator, begins with a random backoff countdown during which the node does not listen to the medium. Once the countdown 


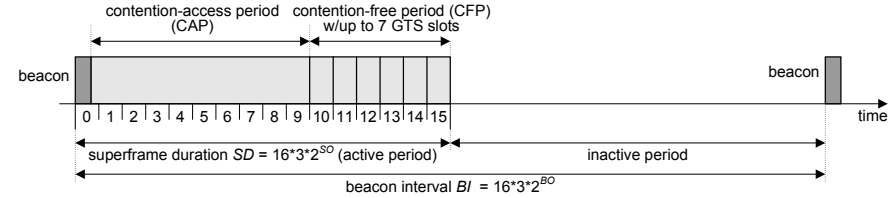

Fig. 2. The composition of the superframe in an IEEE 802.15.4 network (adapted from [20]).

goes to zero, the node listens to the channel during two unit backoff periods. If the medium is idle during that time, transmission may begin; otherwise, a new backoff countdown is initiated. Acknowledgment packets are optional and must be explicitly requested by the sender. In the downlink direction, the coordinator first announces the presence of a packet in the beacon frame. However, the packet is not sent until explicitly requested by the intended recipient. All these transmissions use CSMA-CA medium access to send data, and they take place during the so-called Contention Access Period (CAP).

Optionally, a part of the active portion of the superframe may be used for contention-free access, hence it is referred to as the Contention-Free Period (CFP). A node that wants exclusive access to the medium sends a request during the $\mathrm{CAP}$ to the coordinator (or $\mathrm{CH}$ node, in our case) specifying the duration of exclusive access and the direction (to or from the node). If the coordinator decides to grant access, the allocation is announced in the next beacon frame. The part of CFP granted to a single node for exclusive access is referred to as a guaranteed time slot (GTS); there can be at most seven such slots in a superframe. GTSs are deallocated on explicit request from a node, automatically upon expiry of exclusive access, or by the coordinator if some time needs to be freed in order to maintain the duration of the CAP above the minimum prescribed by the standard [20].

More details on the various aspects of IEEE 802.15.4 operation can be found in the standard [20].

\section{ENERGY-EFFICIENT CLUSTERING}

Initial clustering is performed using any of the available algorithms based on network topology, such as those described in [4], [12]. For simplicity, we assume that a certain number of nodes are initialized as $\mathrm{CHs}$, although a randomized scheme similar to LEACH [17] could also be used. First, the designated $\mathrm{CH}$ nodes begin broadcasting beacons to other nodes. Non- $\mathrm{CH}$ nodes detect the nearest $\mathrm{CH}$ node using Received Signal Strength Indicator (RSSI) or other similar measure, and request to join its cluster. The request includes addresses of other $\mathrm{CH}$ nodes that the node has heard and the corresponding RSSI. Clustering performed in this manner should result in clusters of similar size. Should the clusters end up with widely varying member counts, the BS can instruct the $\mathrm{CH}$ nodes to re-distribute the non- $\mathrm{CH}$ nodes among clusters in order to reduce or, preferably, eliminate the variation.

Once the cluster membership stabilizes, $\mathrm{CH}$ nodes pass the membership information to the $\mathrm{BS}$, which performs channel allocation and informs the $\mathrm{CH}$ nodes accordingly. Channel allocation should aim to minimize inter-cluster interference whilst keeping the cluster membership as uniform as possible [32]. Finally, $\mathrm{CH}$ nodes inform their cluster members about the new channel; they all switch to the new channel and begin normal operation.

Initial phase of the protocol takes place on a dedicated, preset channel known as the common control channel (CCC). A dedicated control channel is necessary in order to separate communications between the $\mathrm{CH}$ nodes and the $\mathrm{BS}$ from that between the $\mathrm{CH}$ nodes and the ordinary nodes in their respective clusters. When the network operates in the ISM band using direct sequence spread spectrum (DSSS), there is a total of 16 channels available [20]. Channel 26 at $2.480 \mathrm{GHz}$ is best suited for use as the CCC, as it is least likely to suffer from interference from WiFi networks in the vicinity [39]. The other 15 channels may be assigned to individual clusters.

Another piece of information sent by the BS to the $\mathrm{CH}$ nodes is their allocated portion of the total rate of information flow, often referred to as sensing reliability $R$ [2], [34]. The $\mathrm{CH}$ nodes use the information about the sensing reliability required from that cluster to calculate the mean duration of node sleep and broadcast it to the members of their respective clusters.

Each node then chooses its own sleep time as a randomly generated number from a selected probability distribution. Mean value of the distribution is set to the mean sleep duration. Randomization attempts to minimize the probability that two or more nodes attempt to send their data at the same time [26].

When a node wakes up, it checks to see if there is sensing data to send to its $\mathrm{CH}$; if there is a packet, the node will send it to its $\mathrm{CH}$ and go to sleep immediately upon receiving a positive acknowledgment. Otherwise, the node immediately selects a new sleep period and goes to sleep again.

The $\mathrm{CH}$ node must subsequently deliver the collected data to the BS. To this end, it will leave its designated channel during the inactive portion of the superframe, switch (i.e., hop on) to the CCC, send the data, then hop back to the cluster channel and resume the $\mathrm{CH}$ role.

Let us now review the communication cost of access modes provided by the IEEE 802.15.4 standard from the perspective of energy expenditure; a detailed analytical model can be found in the Online Supplement.

\section{A. Communication cost of CSMA-CA access}

The default access mode in IEEE 802.15.4 networks is CSMA-CA, the timing diagram of which is shown in Fig. 3(a). The node that awakens with sensing data to send will first synchronize with the beacon emitted by the $\mathrm{CH}$. Since the node awakens at a random time within the beacon interval $B I$ and these two events are independent, we may assume that the distribution of wake-up time within the beacon interval is uniform. As a result, the average synchronization time is onehalf of the beacon interval.

The node then sends its data in the uplink direction. The time needed to finish the transmission successfully is a random variable which depends on the chosen initial value for the random backoff countdown. Once the transmission succeeds, the node may go back to sleep again. Most transmissions will 


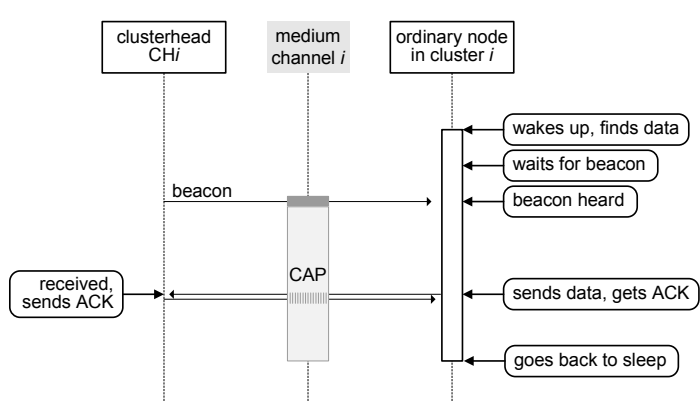

(a) CSMA-CA access

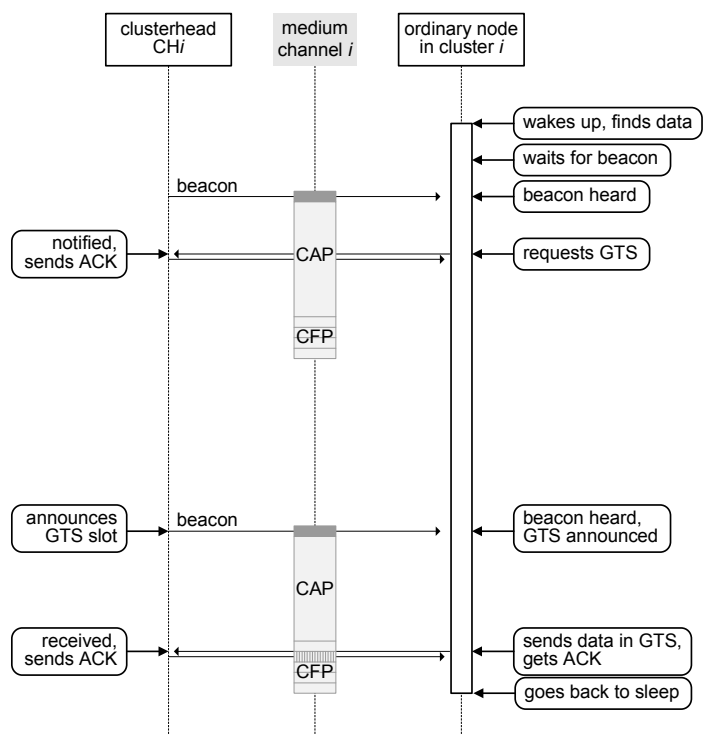

(b) TDMA access with an explicit GTS request.

Fig. 3. Access modes for intra-cluster communication.

succeed in a single superframe, therefore the total time interval during which a node must remain active will be the sum of one-half of the beacon interval and one superframe duration.

Note that beacon interval $B I$ must be strictly longer than the superframe duration $S D$ (hence, $B O>S O$ ) because the $\mathrm{CH}$ node needs time to switch to the $\mathrm{CCC}$ and deliver the data to the BS.

\section{B. Communication cost of TDMA access with explicit GTS requests}

Transmission using TDMA access via GTS is shown in Fig. 3(b). As before, the node that awakens with sensing data to send need to synchronize with the beacon emitted by the $\mathrm{CH}$, and the average synchronization time is one-half of the beacon interval.

As the $\mathrm{CH}$ does not know which nodes will be active at which time, GTS slots are allocated on request only. A GTS slot request packet must be sent to the $\mathrm{CH}$ node, which announces the allocation in the next beacon frame; only then can the node send its data frame in the allocated slot. Therefore, TDMA access requires the node to be active for one full beacon interval longer than when CSMA-CA access is used. In case $S O=0, B O=1$, which corresponds to the shortest possible beacon interval and superframe duration, this translates into about $40 \%$ more time spent in active state when using TDMA access. Larger values of $S O$ and $B O$ would reduce the relative advantage of CSMA-CA at the expense of increasing the absolute values of active time.

As in the case of CSMA-CA access, transmission of the GTS request may fail due to noise and interference, as well as because of a collision with a similar request sent by another node. While data frame transmission cannot suffer a collision - other nodes are forbidden to use that slot - it can still fail due to noise and interference. In both cases, transmission will be required until successful.

\section{On access mechanism for $\mathrm{CH}$-to-BS communications}

The last issue to consider is the access mode for $\mathrm{CH}$-toBS transmissions. The traffic on the CCC is equal to the sum of data traffic of all clusters. However, the number of clusters (and their $\mathrm{CHs)}$ is not too high, and not every $\mathrm{CH}$ node will have data in each superframe due to randomization of sensing node sleep intervals. Therefore, contention will not be a problem, and we can use CSMA-CA access on account of its simplicity.

\section{Other considerations}

In both schemes outlined above, nodes use randomized sleep to conserve energy. The $\mathrm{CH}$ node could also set a fixed schedule in which nodes use deterministic sleep. However, this requires non-negligible computational effort by the $\mathrm{CH}$ node and the need to re-calculate the schedule when a node dies or fails in another way. Also, deterministic sleep could easily lead to prolonged intervals without sensing, which may be disadvantageous in some cases.

We note that explicit GTS requests might be avoided if the coordinator would devise a fixed schedule of GTS slots that includes all nodes, and holds on to it throughout the network lifetime. However, the schedule would span several superframes as no superframe may contain more than seven GTS slots [20]. As a result, nodes have to wait for the proper superframe to send their data - some nodes would get the slot in the first, some in the last superframe. To balance their energy expenditure, the $\mathrm{CH}$ would need to re-arrange the schedule periodically, and schedule updates are also needed to account for nodes' death. As both of these approaches require additional (and non-trivial) computational effort, we have decided to use randomized sleep, and to focus on CSMACA access and TDMA access with explicit GTS requests. The Online Supplement presents a detailed analytical model of network operation in these two scenarios, including the impact of noise and interference as well as of collisions due to concurrent transmissions.

\section{Cluster-Head handover}

$\mathrm{CH}$ nodes use much more energy than the non- $\mathrm{CH}$ nodes, as outlined in Section I. To reduce or, hopefully, eliminate the imbalance, the $\mathrm{CH}$ role should be rotated among all the nodes in the cluster. Namely, over the period of its service 
(hereafter referred to as a round), the current $\mathrm{CH}$ node records the number of packets received by each ordinary node, as well as the current energy level reported by the node itself. When the number of packets received from a single node reaches a predefined handover threshold, the current $\mathrm{CH}$ node hands over the $\mathrm{CH}$ duty to the node with the highest remaining energy. In this manner, the $\mathrm{CH}$ duty and the associated energy expenditure are evenly distributed amongst all nodes in the cluster [17].

\section{A. The handover protocol}

The actual protocol for $\mathrm{CH}$ handover is adapted to the operation of the IEEE 802.15.4 network. Once the threshold packet count is reached, the current $\mathrm{CH}$ finds the node with the highest reported energy. When this node wakes up and sends a packet, the current $\mathrm{CH}$ sends a special acknowledgment labeled 'handover ACK.' Note that the node with the highest energy at the time of reaching the threshold may not end up as the new $\mathrm{CH}$ : other nodes, possibly with higher energy level, may wake up before it. In this case, the node with higher energy is immediately assigned to the $\mathrm{CH}$ duty in the next round. It is worth noting that this procedure works under both CSMA-CA and TDMA access modes.

When a node received a handover ACK instead of the ordinary one, it sends a notification - effectively, an acknowledgment packet - in the uplink direction. It then begins to act as the $\mathrm{CH}$ : i.e., it emits its own beacons and receives the packets from other nodes. The previous $\mathrm{CH}$ simply reverts to the role of the ordinary node. The cluster-head handover protocol are schematically shown in Fig. 4. Note that the transition from one $\mathrm{CH}$ to another is allowed by the IEEE 802.15.4 standard [20], since the beacon frame contains the PAN id through which ordinary nodes identify the cluster. The source node id, which corresponds to the $\mathrm{CH}$ node that sent the beacon frame, may be ignored by the upper layers of the protocol stack.

The solution described above relies on the remaining energy of nodes for $\mathrm{CH}$ selection, similar to [11], [15], [17], [40], but does not require that nodes to exchange information about their energy levels in order to select the next $\mathrm{CH}$. Instead, the $\mathrm{CH}$ reaches the decision locally on the basis of the information it has collected in the course of its service. As cluster membership is fixed, there is no re-clustering which consumes energy and imposes a 'dead time' during which the WSN is not operational. Consequently, the proposed solution is more energy efficient in terms of both communication and computation.

\section{B. Dealing with node and $\mathrm{CH}$ failures}

We also need to consider how to deal with node failures, in particular nodes' death that occur due to battery exhaustion.

If the $\mathrm{CH}$ does not hear a node for a predefined timeout, it may assume that it has died. However, the $\mathrm{CH}$ need not take any action except for re-calculating the mean sleep time and announcing it to the other nodes.

If the current $\mathrm{CH}$ dies, ordinary nodes will notice there is no cluster superframe to synchronize with. After a predefined

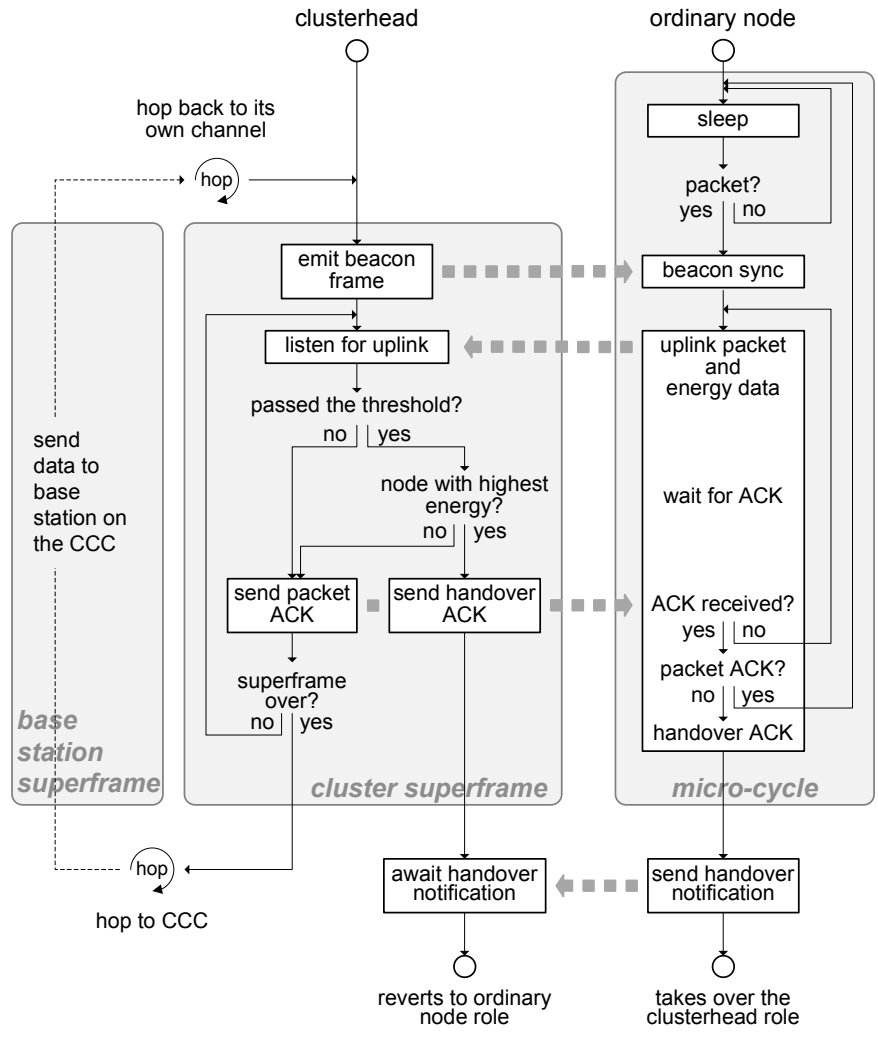

Fig. 4. Cluster operation and cluster-head handover protocol.

timeout, they can switch to the $\mathrm{CCC}$ and report to the BS. (Note that the BS will also note the absence of the traffic from the affected cluster.) The BS can initiate re-clustering of the entire network, but this procedure is lengthy and requires that all nodes are informed so that they san be awake. A cheaper (i.e., more energy efficient) solution is to simply assign the orphaned nodes to a different cluster. This approach is much faster than re-clustering, does not incur any dead time, and no special action is needed from the $\mathrm{CH}$ of their new cluster aside from re-calculating the mean sleep time and announcing it to the nodes in its cluster.

However, nodes' death will reduce the amount of traffic from the cluster to the BS, while re-assignment of orphaned nodes will increase the traffic from their new cluster. Either way, the BS should monitor the amount of traffic received from each cluster, typically averaged over a suitable time window. If the total reliability $R$ drops below a predefined threshold, the BS will re-calculate the required values of percluster reliability $R_{i}$ and inform the current $\mathrm{CHs}$ accordingly. As the CHs appear often on the CCC to deliver their data, they can learn about the adjustment rather quickly. In this manner, the overall load will be uniformly distributed over all non- $\mathrm{CH}$ nodes in the network.

A handover might fail because the node with the highest remaining energy dies before reporting to the current $\mathrm{CH}$. The problem can be easily dealt with by introducing a timeout to make the decision to handover the $\mathrm{CH}$ duty, possibly expressed in the number of received packets after the threshold is reached. Fortunately, the likelihood of a failure of this type 
is small, except maybe near the end of the network lifetime when nodes begin to die in larger numbers.

\section{On node homo- and heterogeneity}

Our approach does assume that nodes are homogeneous, but heterogeneity can be accommodated with ease. First, node heterogeneity due to different size of energy source is accommodated automatically in the cluster-head handover protocol. Second, nodes that generate more (or less) traffic than the average, perhaps because they host a different type of sensor, can also be accommodated with a bit of extra effort. Namely, such nodes are free to send sensing data as often as necessary, but they need to register as such during the initial clustering. (Registration is needed since the $\mathrm{CH}$ cannot make any decisions based on history of received data because of randomness of the traffic and comparatively short duration of the round of $\mathrm{CH}$ duty.) Upon registration, the $\mathrm{CH}$ node would set a different handover threshold for each such node. During the handover itself, the information about different thresholds will be passed on from one $\mathrm{CH}$ to the next, which is not hard to accomplish in the context of IEEE 802.15.4 networks.

\section{PERFORMANCE EVALUATION}

We now present numerical results obtained by solving the analytical model presented in the Online Supplement using Maple 13 from MapleSoft, Inc. [25]. We considered an IEEE 802.15.4 network operating in the ISM band at $2.4 \mathrm{GHz}$, with raw data rate $250 \mathrm{kbps}$. The effect of noise is modeled with $B E R=10^{-4}$. Superframe size and beacon interval are set to 48 and 96 unit backoff periods, respectively, by setting $S O=0$ and $B O=1$. The network consists of $n=400$ nodes powered with two AA batteries with the energy of $E_{\text {bat }}=10260 \mathrm{~J}$ each which may be obtained with NickelMetal-Hydride (NiMH) or Lithium-Ion batteries, according to tables at http://www.allaboutbatteries.com/Energy-tables.html. Using the data for Chipcon CC2420 2.4GHz RF Transceiver [38], energy consumption per unit backoff period was set to $\omega_{s}=18.2 n \mathbf{J}, \omega_{r}=17.9 \mu \mathrm{J}$ and $\omega_{t}=15.8 \mu \mathrm{J}$, during sleep, receiving and transmitting at $0 \mathrm{dBm}$, respectively.

We assume that sensing packets have a length of $\bar{D}_{d}=12$ backoff periods, or about 120 bytes; two bytes in each sensing packet are used to report the current energy level of a node to the $\mathrm{CH}$. Sensing packets arrive according to a Poisson process with the arrival rate of one packet per node per second, which serves to ensure the required sensing reliability. Each node has a buffer size of $L=2$ packets; larger buffers are unnecessary due to the use of randomized sleep.

We have conducted a number of experiments, using sensing reliability $R$, number of clusters $N_{c}$, and handover threshold $N_{\mu}$ as independent variables. Sensing reliability was varied in the range from 25 to 250 packets per second in steps of 25 , handover threshold was varied from 3 to 30 packets in steps of 3 , and number of clusters was varied from 1 to 28 in steps of 3 . The case where $N_{c}=1$ - effectively, a nonclustered network - allows us to gauge the impact of clustering on network lifetime.
We use our clustering scheme with both CSMA-CA and TDMA access. As most of the approaches reviewed in Section II use TDMA, and very few of them use IEEE 802.15.4, we believe that this is a fair representation of the performance of those protocols, if they had been implemented using IEEE 802.15.4 technology. In fact, it is even more favorable to those protocols that use re-clustering and $\mathrm{CH}$ election by all nodes, since these features (which actually degrade performance) are omitted in our evaluation.

\section{A. Characterizing the network lifetime}

The focus of our evaluation is the network lifetime, shown in Fig. 5 for CSMA-CA and TDMA access on the left and right, respectively.

As can be seen, when sensing reliability is low, smaller number of clusters results in longer lifetimes, due to the difference in energy expenditure between $\mathrm{CH}$ and non- $\mathrm{CH}$ nodes: fewer clusters mean fewer $\mathrm{CH}$ nodes and more nodes per cluster. When the reliability is low, fewer $\mathrm{CH}$ nodes means less energy expenditure, while non- $\mathrm{CH}$ nodes sleep longer and their transmissions rarely collide.

However, when the number of clusters increases, the number of ordinary, non- $\mathrm{CH}$ nodes in each cluster decreases. Smaller number of nodes leads to a very low collision rate and reduces the time that a non- $\mathrm{CH}$ node spends in an active state. Since the energy consumption is dominated by the $\mathrm{CH}$ nodes (of which there are many), the impact of reliability on network lifetime is diminished almost to the point of being negligible, as witnessed by the flattening of the surfaces observed in the diagrams. This effect can be observed under both CSMA-CA and TDMA access; it may be of interest in cases where the number of clusters is determined on the basis of other factors, rather than just energy efficiency.

At low values of reliability (i.e., when $R<100$ packets per second), non-clustered network (the lines at the far right corner in Figs. 5(a) and 5(b), where $N_{c}=1$ ) achieves longest lifetime values. However, when the reliability increases, collisions also increase [27], [28] and network lifetime drops rapidly. As a result, non-clustered network is virtually unusable at reliability values over $R=150$ to 200 packets per second.

In general, CSMA-CA access results in longer network lifetime than TDMA access. As discussed in Section IV-B, this is due to the high overhead of TDMA access which requires at least two packets in two successive superframes to transmit a single data packet in the uplink direction, as opposed to one data packet in a single superframe required under CSMA-CA access.

Figs. 5(c) and 5(d) indicate that mean network lifetime is virtually independent of the value of handover threshold, for both access modes. In case of CSMA-CA access, the resulting dependency on sensing reliability is nearly linear. In case of TDMA access, mean network lifetime is initially only slightly smaller than that under CSMA-CA access. However, when sensing reliability increases, it drops more rapidly, reaching about two-thirds of it at $R=250$ packets per second. 


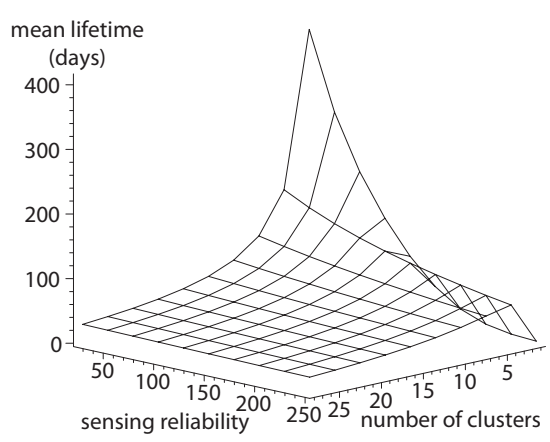

(a) CSMA-CA access, handover threshold of $N_{\mu}=12$ packets.

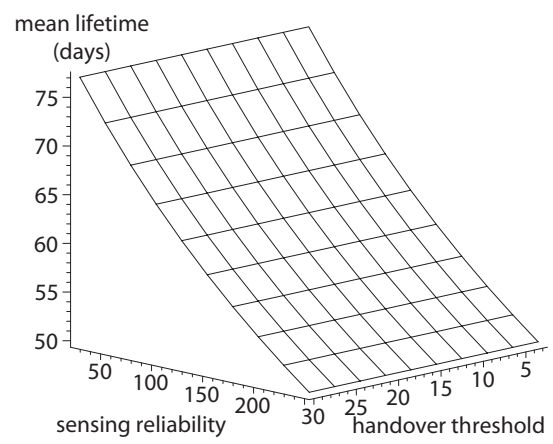

(c) CSMA-CA access, $N_{c}=10$ clusters.

Fig. 5. Mean network lifetime in days.

\section{B. Improving network lifetime through $\mathrm{CH}$ node sleep}

The results presented above clearly indicate that the main obstacle to achieving longer network lifetime is the excessive energy expenditure of the nodes currently acting as CHs. An obvious remedy appears to be to send $\mathrm{CH}$ nodes to sleep as well. Yet if a $\mathrm{CH}$ node goes to sleep for a protracted time, ordinary nodes in its cluster would have to wait too long to synchronize to the beacon and waste too much energy in the process, thus offsetting any energy savings resulting from $\mathrm{CH}$ node sleep. Furthermore, long periods of $\mathrm{CH}$ node sleep would increase the likelihood that two or more ordinary nodes attempt to send their data in the same superframe, and the increased collision probability would lead to even higher energy expenditure.

Fortunately, the CSMA-CA protocol implemented in IEEE 802.15.4 allows for a simple mechanism that reduces the active time of the $\mathrm{CH}$ nodes. Namely, when an ordinary node wakes up, it waits for the beacon, executes a backoff countdown initially chosen in the range from 0 to 7 unit backoff periods, performs two CCA checks, each lasting a single unit backoff period, and then attempts to send its data [20]. Therefore, the first transmission attempt in a superframe, if any, must occur in the first 10 unit backoff periods after the beacon frame.

Hence, if there is no transmission in the first 10 unit backoff periods after the beacon, the $\mathrm{CH}$ node may safely conclude there are no nodes awake to send data in the current superframe. It can, then, safely go to sleep until the beginning of the next superframe, as shown in Fig. 6.

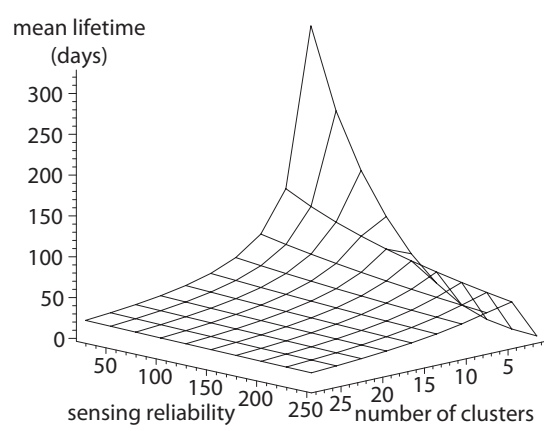

(b) TDMA access with explicit GTS requests, handover threshold $N_{\mu}=12$ packets.

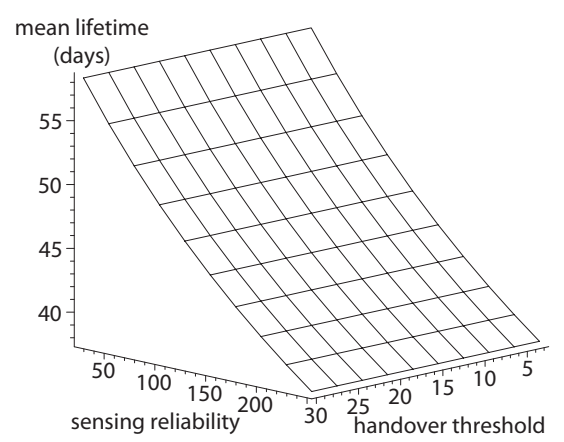

(d) TDMA access with explicit GTS requests, $N_{c}=12$ clusters.

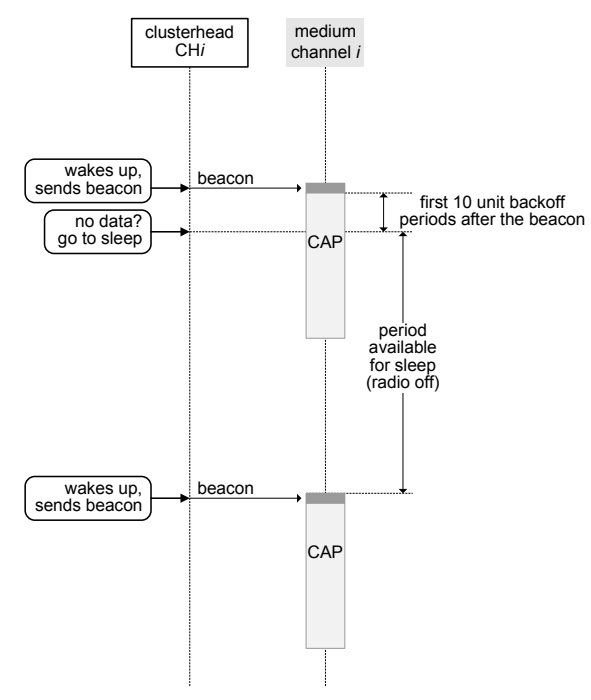

Fig. 6. $\mathrm{CH}$ node sleep.

The resulting network lifetime is shown in Fig. 7. We have used the same network parameters as above. These diagrams contain the results obtained from the analytical model presented in the Online Supplement as well as those obtained through a discrete event simulator built using the Petri net simulation engine Artifex from RSoftDesign, Inc. [33]. As can be seen, this simple modification extends the lifetime of the network considerably. The difference in network 


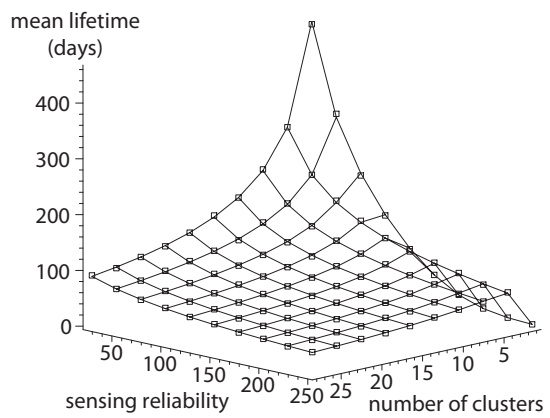

(a) CSMA-CA access, handover threshold of $N_{\mu}=12$ packets

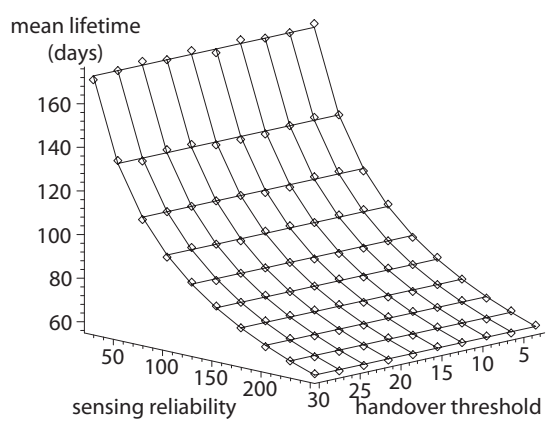

(c) CSMA-CA access, $N_{c}=12$ clusters.

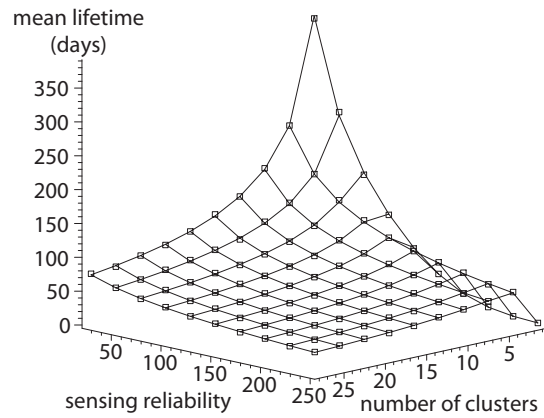

(b) TDMA access with explicit GTS requests, handover threshold of $N_{\mu}=12$ packets.

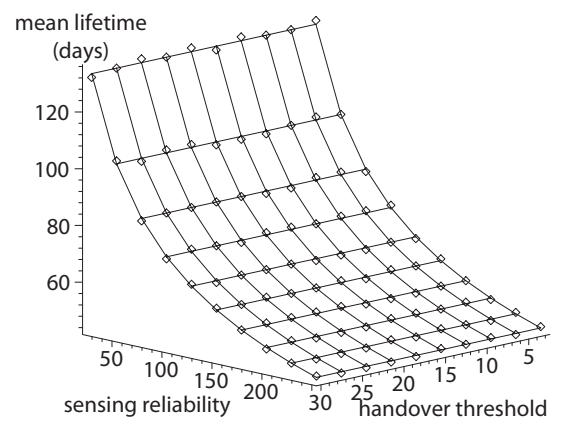

(d) TDMA access with explicit GTS requests, $N_{c}=12$ clusters.

Fig. 7. Mean duration of network lifetime when $\mathrm{CH}$ nodes sleep. Lines denote analytical results, diamonds denote simulation results.

lifetime between CSMA-CA and TDMA access is even greater than before, as improvements obtained through opportunistic $\mathrm{CH}$ node sleep are actually larger under CSMA-CA access. It is worth noting that there is very little improvement in the performance of the non-clustered network $\left(N_{c}=1\right)$, esp. under TDMA access, which can be expected as there are no $\mathrm{CH}$ nodes to benefit from opportunistic sleep.

We also note quite good agreement between analytical and simulation results, which confirms the validity of our model.

\section{The balance of nodes' lifetime}

To investigate the degree of balancing achieved through the use of randomized sleep and cluster-head rotation, we have calculated the coefficient of variation and skewness of node lifetime in the network where $\mathrm{CH}$ nodes sleep in the aforementioned manner. The results are shown in Fig. 8 for CSMA-CA access mode only due to its superior performance compared to TDMA access mode.

As can be seen, both the coefficient of variation and skewness are well below $1 \%$ throughout the observed range of independent variables, with a single exception which we elaborate below. Such low values can be explained as follows. Each node sleeps for a random time several times during a single round. A round consists of a number of random sleep intervals. Each node undergoes several rounds acting as an ordinary sensing node between successive rounds in which it takes on the $\mathrm{CH}$ duty. During its lifetime, a node serves several times as the $\mathrm{CH}$. Randomization thus exists at several different levels and the node lifetime is, thus, a sum of a number of independent, identically distributed random variables. According to central limit theorem [13], distribution of such a sum tends toward the normal distribution which has zero skewness and standard deviation inversely proportional to the square of the number of summands.

Values of coefficient of variation close to zero indicate that the variability of nodes' lifetime is extremely low - in other words, the individual nodes' lifetime will be very close to each other, and most nodes will die at the same time. If the mean network lifetime is, say, 100 days (which our approach achieves over a wide range of sensing reliability values), the coefficient of variation of $0.15 \%$ means that the standard deviation of node lifetime is only $\sigma=0.15$ days, or about 3 hours and 36 minutes. Then, about $90 \%$ of the nodes in this network would die within $\pm 1.65 \sigma$ (about 12 hours) about the mean, and $99.9 \%$ nodes will die within $\pm 3.3 \sigma-$ a single day. Thus the network lifetime, defined as the time period in which all nodes initially present in the network are still alive, is optimized in the maximin sense [16].

The exception noted above refers to the fact that both coefficient of variation and skewness of the network lifetime exhibit notable increase at higher values of reliability in the case of single-cluster network $\left(N_{c}=1\right)$. This is the consequence of increased collision rate and longer time spent in the active state, both of which are caused by the large number of nodes in the cluster. As a result, individual nodes' lifetime values exhibit larger variability which, in turn, leads to the drop in network lifetime observed in Fig. 7(a) above. 


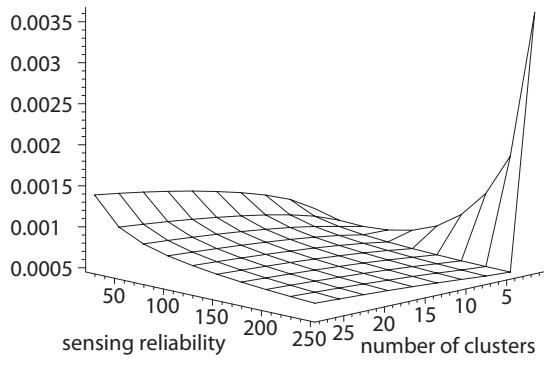

(a) Coefficient of variation, handover threshold of $N_{\mu}=12$ packets.

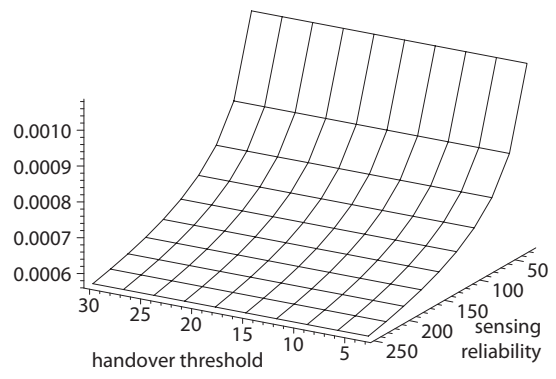

(c) Coefficient of variation, $N_{c}=12$ clusters.

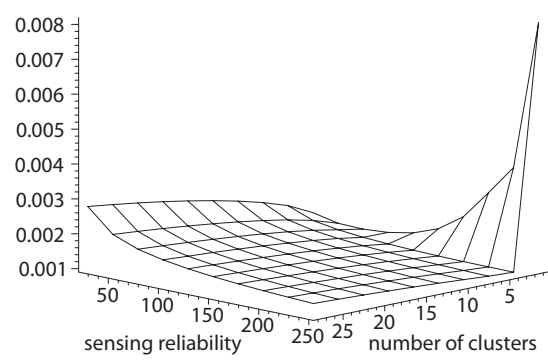

(b) Skewness, handover threshold of $N_{\mu}=12$ packets.

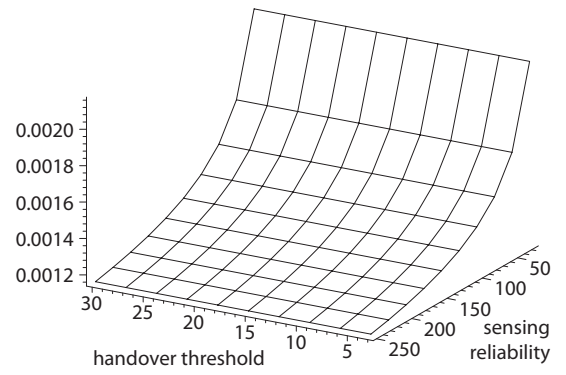

(d) Skewness, $N_{c}=12$ clusters.

Fig. 8. Coefficient of variation and skewness of network lifetime under CSMA-CA access.

\section{CONCLUSION}

We have described a novel energy-efficient approach to clustering, fully integrated with the IEEE 802.15.4 low data rate WPAN standard. The approach uses probabilistic sleep of ordinary nodes, opportunistic sleep of cluster-head nodes, and efficient cluster-head rotation with no overhead and zero dead time. By eliminating nearly all sources of inefficiency and unfairness, our approach achieves extremely good balancing of nodes' lifetime and, thus, optimizes the network lifetime. In conjunction with CSMA-CA access, which achieves considerably longer node and network lifetime compared to TDMA access, the proposed approach offers superior performance compared to other existing algorithms that employ frequent re-clustering and/or TDMA scheduling.

Our future work will focus on refining the approach and on verifying its performance in practice. We also plan to incorporate changes needed to accommodate sensors with heterogeneous traffic and different power sources.

\section{REFERENCES}

[1] H. Abusaimeh and S.-H. Yang. Dynamic cluster head for lifetime efficiency in WSN. Int. J. of Automation and Computing, 6(1):48-54, 2009.

[2] I. F. Akyildiz, W. Su, Y. Sankarasubramaniam, and E. Cayirci. Wireless sensor networks: a survey. IEEE Communications Magazine, 40(8):102114, August 2002.

[3] G. Anastasi, M. Conti, M. Di Francesco, and V. Neri. Reliability and energy efficiency in multi-hop IEEE 802.15. 4/ZigBe wireless sensor networks. In IEEE Symp. Computers Communications (ISCC 2010), pages 336-341, 2010.

[4] S. Bandyopadhyay and E. J. Coyle. An energy efficient hierarchical clustering algorithm for wireless sensor networks. In Proc. INFOCOM 2003, volume 3, pages 1713-1723, 2003.

[5] X. Bian, X. Liu, and H. Cho. Study on a cluster-chain routing protocol in wireless sensor networks. In Proc. ChinaCom 2008, pages 964-968, Aug. 2008.
[6] O. Boyinbode, H. Le, A. Mbogho, M. Takizawa, and R. Poliah. A Survey on Clustering Algorithms for Wireless Sensor Networks. In Proc. 13th Int. Conf. Network-Based Inf. Syst. (NBiS 2010), pages 358-364, Sept. 2010.

[7] C. Buratti, A. Ferri, and R. Verdone. An ieee 802.15. 4 wireless sensor network for energy efficient buildings. In The Internet of Things, pages 329-338. Springer, 2010.

[8] G. Chen, F. Nocetti, J. Gonzalez, and I. Stojmenovic. Connectivity based $k$-hop clustering in wireless networks. In Proc. 35th Ann. Hawaii Int. Conf. Syst. Sci. HICSS 2002, pages 2450-2459, Jan. 2002.

[9] S. C. Ergen and P. Varaiya. TDMA scheduling algorithms for wireless sensor networks. Wirel. Netw., 16(4):985-997, May 2010.

[10] J. Frolik. QoS control for random access wireless sensor networks. In Proc. WCNC 2004, Atlanta, GA, Mar. 2004.

[11] S. Gamwarige and C. Kulasekere. Optimization of cluster head rotation in energy constrained wireless sensor networks. In Proc. IFIP Int. Conf. Wireless Opt. Comm. Networks (WOCN'07), Singapore, 2007.

[12] S. Ghiasi, A. Srivastava, X. Yang, and M. Sarrafzadeh. Optimal energy aware clustering in sensor networks. Sensors, 2(7):258-269, 2002.

[13] G. R. Grimmett and D. R. Stirzaker. Probability and Random Processes. Oxford University Press, Oxford, UK, 2nd edition, 1992.

[14] V. C. Gungor, B. Lu, and G. P. Hancke. Opportunities and challenges of wireless sensor networks in smart grid. IEEE Trans. on Industrial Electronics, 57(10):3557-3564, 2010.

[15] M. Handy, M. Haase, and D. Timmermann. Low energy adaptive clustering hierarchy with deterministic cluster-head selection. In Proc. MWCN 2002, pages 368-372, 2002.

[16] Minimax principle. In M. Hazewinkel, editor, Encyclopedia of Mathematics. Springer Verlag, 2001.

[17] W. Heinzelman, A. Chandrakasan, and H. Balakrishnan. Energy-efficient communication protocol for wireless microsensor networks. In Proc. 33rd Ann. Hawaii Int. Conf. Syst. Sci. HICSS 2000), page 10 pp. vol.2, Jan. 2000

[18] W. Heinzelman, A. Chandrakasan, and H. Balakrishnan. An applicationspecific protocol architecture for wireless microsensor networks. IEEE Trans. on Wireless Communications, 1(4):660-670, Oct. 2002.

[19] J. Hong, J. Kook, S. Lee, D. Kwon, and S. Yi. T-LEACH: The method of threshold-based cluster head replacement for wireless sensor networks. Information Systems Frontiers, 11(5):513-521, Nov. 2009.

[20] IEEE. IEEE Standard for Local and metropolitan area networks - Part 15.4: Low-Rate Wireless Personal Area Networks (LR-WPANs). IEEE Standard 802.15.4-2011, IEEE, New York, NY, 2011. 
[21] R. Iyer and L. Kleinrock. QoS control for sensor networks. In Proc. ICC'03, volume 1, pages 517-521, Anchorage, AK, May 2003.

[22] N. Johansson, U. Korner, and L. Tassiulas. A distributed scheduling algorithm for a Bluetooth scatternet. In Int. Teletraffic Congress, 2001.

[23] J. Kim, J. Lee, and K. Rim. 3DE: selective cluster head selection scheme for energy efficiency in wireless sensor networks. In Proc. PETRA '09, pages 33:1-33:7, 2009.

[24] J. Ko, C. Lu, M. B. Srivastava, J. A. Stankovic, A. Terzis, and M. Welsh. Wireless sensor networks for healthcare. Proceedings of the IEEE, 98(11):1947-1960, 2010.

[25] Maplesoft, Inc. Maple 13. Waterloo, ON, Canada, 2009.

[26] J. Mišić, S. Shafi, and V. B. Mišić. Maintaining reliability through activity management in an 802.15.4 sensor cluster. IEEE Transactions on Vehicular Technology, 55(3):779-788, May 2006.

[27] J. Mišić, S. Shafi, and V. B. Mišić. Performance of beacon enabled ieee 802.15.4 cluster with downlink and uplink traffic. IEEE Transactions on Parallel and Distributed Systems, 17(4):361-377, Apr. 2006.

[28] J. Mišić and V. Mišić. Wireless Personal Area Networks: Performance, Interconnections and Security with IEEE 802.15.4. John Wiley \& Sons, 2008.

[29] S. Naeimi, H. Ghafghazi, C.-O. Chow, and H. Ishii. A Survey on the Taxonomy of Cluster-Based Routing Protocols for Homogeneous Wireless Sensor Networks. Sensors, 12(6):7350-7409, 2012.

[30] M. Noori and M. Ardakani. Lifetime Analysis of Random EventDriven Clustered Wireless Sensor Networks. IEEE Trans. on Mobile Computing, 10(10):1448-1458, 2011

[31] S. Olariu and I. Stojmenovic. Design guidelines for maximizing lifetime and avoiding energy holes in sensor networks with uniform distribution and uniform reporting. In Proc. INFOCOM 2006, pages 1-12, Barcelona, Spain, 2006.

[32] T. Rappaport. Wireless communications: Principles \& Paractice. Prentice Hall, Upper Saddle River, NJ 07458, 1996.

[33] RSoft Design. Artifex v.4.4.2. RSoft Design Group, Inc., San Jose, CA, 2003.

[34] Y. Sankarasubramaniam, Ö. B. Akan, and I. F. Akyildiz. ESRT: eventto-sink reliable transport in wireless sensor networks. In Proc. 4th ACM MobiHoc, pages 177-188, Annapolis, MD, June 2003.

[35] R. Srivathsan, S. Siddharth, R. Muthuregunathan, R. Gunasekaran, and V. Uthariaraj. Enhanced Genetic algorithm for solving broadcast scheduling problem in TDMA based wireless networks. In Proc. COMSNETS 2010, pages 1-10, Jan. 2010.

[36] I. Stojmenović, editor. Handbook of Sensor Networks: Algorithms and Architectures. John Wiley \& Sons, 2005.

[37] I. Stojmenovic, M. Seddigh, and J. Zunic. Dominating Sets and Neighbor Elimination-Based Broadcasting Algorithms in Wireless Networks. IEEE Trans. Parallel Distrib. Syst., 13(1):14-25, Jan. 2002.

[38] Chipcon CC2420 Datasheet: 2.4 GHz IEEE 802.15.4 / ZigBee-Ready RF Transceiver (Rev. B), Feb. 2012.

[39] G. Thonet, P. Allard-Jacquin, and P. Colle. ZigBee-WiFi coexistence. white paper and test report, Schneider Electric, Grenoble, France, 2008

[40] Y. Wu, Z. Chen, Q. Jing, and Y.-C. Wang. Leno: Least rotation nearoptimal cluster head rotation strategy in wireless sensor networks. In Proc. Adv. Inf. Networking and Applications (AINA 2007), pages 195201, Niagara Falls, ON, Canada, 2007.

[41] O. Younis and S. Fahmy. HEED: a hybrid, energy-efficient, distributed clustering approach for ad hoc sensor networks. IEEE Trans. on Mobile Computing, 3(4):366-379, 2004.

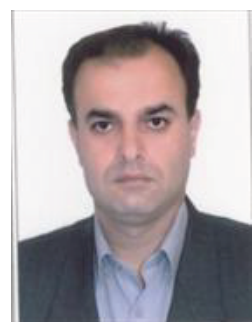

Hamidreza Tavakoli is currently pursuing his $\mathrm{PhD}$ degree in Electrical Engineering at the Iran University of Science and Technology and Ryerson University in Toronto, Canada. His research interest are in wireless sensor networks and green communications technology.

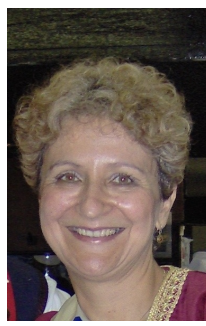

Jelena Mišić (M91, SM08) Professor of Computer Science at Ryerson University in Toronto, Ontario, Canada. She has published over 90 papers in archival journals and more than 120 papers at international conferences in the areas of wireless networks, in particular wireless personal area network and wireless sensor network protocols, performance evaluation, and security. She serves on editorial boards of IEEE Transactions on Vehicular Technology, Computer Networks, Ad hoc Networks, Security and Communication Networks, Ad Hoc \& Sensor Wireless Networks, Int. Journal of Sensor Networks, and Int. Journal of Telemedicine and Applications. She is a Senior Member of IEEE and Member of ACM.

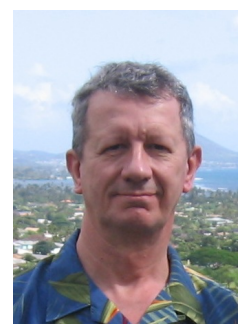

Vojislav B. Mišić is Professor of Computer Science at Ryerson University in Toronto, Ontario, Canada. $\mathrm{He}$ received his $\mathrm{PhD}$ in Computer Science from University of Belgrade, Serbia, in 1993. His research interests include performance evaluation of wireless networks and systems and software engineering. $\mathrm{He}$ has authored or co-authored six books, 20 book chapters, and over 200 papers in archival journals and at prestigious international conferences. $\mathrm{He}$ is a Senior Member of IEEE, and member of ACM and AIS

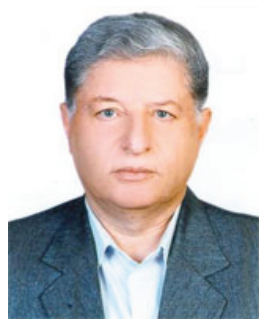

Majid Naderi has received his $\mathrm{PhD}$ in Electronic Engineering from University of Kent, Canterbury, England. He is an Associate Professor of Electrical Engineering at the Iran University of Science and Technology. His research interests include parallel processing, cryptography and secure systems, and design of digital systems. 